\title{
From traditional transactions to B2B: a contract theoretical analysis
}

\author{
María Verónica Alderete \\ CONICET- Institute of Economics, \\ Universidad Nacional del Sur, Department of Economics, malderete@uns.edu.ar \\ Received 17 May 2010; received in revised form 3 September 2010; accepted 15 October 2010
}

\begin{abstract}
This paper extends Alderete (2009) model of screening contracts to build electronic commerce between a large firm and a small and medium sized enterprise (SME) supplier. The large firm (principal) must choose among SME suppliers (agents) that differ in costs and utility for the ICT technology (network good). The method is based on Compte and Jehiel (2008), whose main insight is that when several potential candidates compete for the task, the principal will in general improve the performance of her firm by inducing the member candidates to assess their competence before signing the contract (through an appropriate choice of contracts). In the presence of different preferences for the network good, we show that social surplus increases.
\end{abstract}

Key words: Information Economics, Electronic Commerce, Game Theory, Mechanism Design, Small and Medium Sized Enterprises 


\section{Introduction}

Nowadays, firms, both large, and small and medium sized enterprises (SME), are teaming up more than ever to enhance their competitiveness in the marketplace and follow the rapid changes of technological innovation. To achieve technological innovation in information and communication technologies (ICT), firms, especially SME, increasingly rely on a tight interplay with other firms. Effective business to business (B2B) relationships are of core importance for organizations to enhance their own ability to be more competitive in the marketplace.

In today's business environment more and more transactions are mediated between suppliers and customers over the internet. The scope of electronic commerce $(E C)$ applications is limited to the utilization of the Internet as the technological infrastructure to communicate, distribute and conduct information exchange and transactions with business partners and to carry out transactions and interactions that affect existing business relationships or preexisting contractual relations between trading partners. It is now universally recognized that B2B EC possesses the largest potential in the EC segment, which is supported by a growing trend of companies incorporating one or more of its business initiatives in e-procurement, Customer Relationship Management, online sales and Supply Chain Management [8].

EC is not "one simple or single innovation" that a firm decides whether or not to adopt, but should be considered as consisting of a number of combinations of innovations of varying complexity and sophistication on a continuum requiring lesser to greater levels of commitment. Unlike traditional information systems, EC is an Interorganisational System (IOS), and therefore requires the organization to expand its external electronic links to gain economies of scale and be cost effective [6]. Previous studies have demonstrated that there is a need for "inter-exploitation" between partners through expansion of IOS in order to sustain strategic advantage [2].

According to [20] Internet and e-commerce have the potential to reduce transaction costs and to increase transactions' speed, trust and value at firm level in their value chains. They can reduce inefficiencies from a lack of coordination between a value chain's firms. An empirical study on Spanish firms by [15] argue that Internet use reduces internal coordination costs and transaction costs as a result of the positive relationship between internet use, the degree of vertical integration and technological agreements with suppliers and clients.

Introducing ICT infrastructures capable of sustaining interfirm, indeed network-level, processes requires digitalizing. That means also including into Information Technology (IT) systems the single connections among the involved firms. In other words, network-level IT infrastructures need to have business relationships digitalized, at least partly, as a first step towards digitalizing entire networks. The former requires that the involved firms make an extra effort to understand how their internal activities are related with network-wide processes and which information inputs into IT tools are required from each party [3].

In IT business networks, the value of participating for each firm increases with network size. In the presence of network effects [17], [9], the utility from adoption increases in the number of other adopters that purchase or introduce the innovation. A telephone becomes more valuable to an individual as the total number of telephone users increases. This is a direct network effect and network size approximates the adopter's desire for horizontal compatibility. A DVD player becomes more valuable as the variety of available DVDs increases, and this variety increases as the total number of DVD users increases. This is an indirect network effect. Besides, when network effects are indirect, network size can be a proxy for the availability of complementary components in the technical system. In this case network effects are physical in the sense that they require the hardware and software components of the system to be compatible. In the presence of technical change, as long as backward compatibility is maintained, the network effect ensures that utility from consumption increases. At a specific point in time, network size conveys information to potential adopters about the characteristics of the technology and the payoff from its adoption, which helps firms to make inferences about the opportunities from adoption. This is an example of virtual network effects [11], which can be understood as a particular mechanism for conveying learning spillovers.

Network effects have generally been modeled in a direct sense, even when the effect is thought to operate in an indirect sense, through a complementary good [6]. Following [6] this study considers that firms value being part of a large network, i.e. using an ICT (information and communication technology) business tool that many other firms also use. This is the direct network effect. Firms also value a hardware technology for which there is a wide variety of software available, and more software's firms associate with a hardware technology if more firms use it. This is the indirect network effect. For example, windows operative system is widely used among firm's computers, because a lot of management software (as Electronic Data Interchange, EDI) uses windows, instead of other operative system, as MAC.

Interdependence is a powerful source of positive feedbacks among adopters who take into account both the current network size and its perspective for future growth. Buyer supplier EC means finding a partner with which the firm can establish a bilateral relationship and having the partner undertake relationship-specific investments so that it becomes able to respond electronically to the firm's particular needs. The increasing return properties inherent to network effects then magnify the relative benefits offered, thus triggering positive feedback dynamics. 
There are networks with a leader company, usually a large company that establishes the membership requirements. Large companies that purchase from relatively small vendors can exert power over those vendors in purchasing negotiations. For instance, in 2000, The Harley Davidson Company decided to focus its cost reduction and quality improvement efforts on its information technology infrastructure. Since it had been so successful in working with its suppliers to reduce manufacturing costs and improve quality, Harley Davidson wanted to do the same thing with information technology. By using internet technologies to share information throughout the supply chain, the company hoped to find opportunities for efficiencies and cost reductions at all stages of the process of creating motorcycles. The company decided to standardize forms and procedures and require suppliers to use EDI. For smaller suppliers, the company set up a Web site that had internet EDI capabilities. The smaller suppliers could simply log into the Web site and conduct EDI transactions through their Web browsers [22]. Another example comes from Moen, a firm producer of set of faucets for bathrooms. In October 2000 the firm built his extranet site SupplyNet. This extranet let pieces suppliers to monitor online the situation of Moen's requests of pieces. When Moen changes a request, the supplier receives an email. If a supplier can not attend a request on time, it can notify to Moen immediately so that he can search for the piece in another place. Nowadays, the 40 main suppliers responsible of $80 \%$ of the pieces bought by Moen, use SupplyNet. The result was that the firm saved 3 millions dollars (Business Week Online, 2001). One more example is the Dow Quemical Company, a leader in the use of ecommerce in the chemical industry, resulting in significant cost savings across the value chain. It can conduct an online auction with invited, pre-qualified suppliers. This model allows them to work only with suppliers they already know, who already have been qualified, and who they are already confident that they can do business with. Applying this model through the ChemConnect or the FreeMarkets website, the company has purchased raw materials, as well as ocean freight, heat exchangers, and pipes. It offers increased transaction efficiency, standardized business processes and improved information sharing through the Elemica network. Dow is dedicated to work closely with local suppliers and to educate them about their strategic purchasing requirements and help local suppliers to understand the needs and the potential actions for their businesses which will help them to be globally competitive.

This paper develops a simple principal agent model to explain the development of a buyer-supplier business to business network. It analyses the decision of a large firm about contracting an innovative SME supplier to initiate into business to business. The suppliers are planning to modernize their businesses by incorporating ICT, but they are not able to do it alone, due to financial constraints and technical assistant support needs. Thus, contracting with the large firm is the only way for a SME supplier to introduce an ICT business tool. Contracting problems between a large firm and her SME suppliers limit the contract types design. The large firm searches for a particular supplier partner to build electronic commerce. The study finds that network effects related to technology compatibility increase the social expected surplus.

\section{ICT Adoption at SME Level: Brief Literature Review}

Most SME perceive the challenge of integrating Electronic Commerce (EC) into their business operations as risky, complex, time-consuming, and an expensive initiative. Despite technology facilitating improved business practice in terms of developing electronic markets, electronic data interchange and Internet commerce, many SME have not taken advantage of this mode of business [8]. Besides, there is still uncertainty on the real value that expanding a presence in EC may add to the business.

Yet it should be noted that if EC implementations are successful, the potential benefits to small businesses can include increased sales, improved profitability, increased productivity, reduced costs associated with inventories, procurement and distribution, improved quality of service, and secured competitive positions ([14], [16], and [23]). SME could benefit from cooperative arrangements with other organization and business entities. Joining forces with another organization can allow their business to finance certain services or production functions by sharing expertise, assets, expenses, and risk.

There are many theoretical and empirical contributions to the literature on ICT adoption. Some authors investigate what are the drivers of ICT adoption in SME [12], [18], [29]. In studying individual choices, the literature on technology adoption generally focuses on three sets of determinants: adopters' characteristics, features of the competitive environment, and attributes of the technology [12]. Among adopters' characteristics, firm size, R\&D expenditures, age, and capabilities are usually considered among the main determinants.

The use of IT and EC is often imposed on SME by major customers or suppliers. Such pressure from trading partners plays a critical role in encouraging small firms to adopt IT and EC, as evidenced in previous studies [14], [29], and [30]. The competitiveness of the environment has been found to provide the "push" for SME to deploy their existing capabilities [8]. Firms that fear losing customers or market share may expedite their learning process or expand the use of new systems in order to keep up with what their competitors are doing. The emergence of competitive pressure as a key variable also underscores the need to electronically integrate business operations both internally and externally with trading partners. On the other hand, the selection of suppliers by large firms based just on product price has leaded to numerous disadvantages to suppliers and buyers. These disadvantages have made purchasing departments to change from a price based supplier selection to a broader selection model, in 
which suppliers are no longer selected based solely on prices, but also in aspects such as product quality and warranty [5]

Usually, there are networks dominated by a leading company, often a large firm which sets the rules for being in the network. In the traditional supply chain oriented to mass production, as seen in the vehicle sector, the subcontractors are often "mono-client" and network membership is relatively fluid. In this situation, EC can reduce communication costs and lead times, without altering the vertical power relations within the network. In addition, networks, characterized by leaders can be focused on customized production, with membership depending more on quality, reliability, skill and coordination. Here, EC tools are used for selecting members. Such networks characterize traditional vertical supply chains. Often, forms of ecommerce, such as EDI, are used to select members, bringing rigidity to the network; EDI is mostly adopted in the internal networks of large company leaders and is consequently extended to the external networks of SME suppliers.

When larger firms in supply chains attempt to impose e-business technologies on other members, SME do not have the skill or time to implement all the Intranet applications requested. Firm size also impacts on the level of resources available for investment in information technologies and associated training and education which can inhibit SME adoption. Studies have highlighted that smaller businesses are often less aware of the full potential benefits of ebusiness. Beyond lack of awareness, SME have been shown to exhibit a greater uncertainty of the benefits of IT adoption than larger firms. Despite the apparent benefits of ICT, many SME are unsure of what are the appropriate ICT solutions in which to invest [27].

ICT adoption decisions are highly path-dependent, follow cumulative linear adoption patterns as managers' perceptions of the benefits of technologies significantly depend in previously adopted technologies and equipment. Rogers (see [19]) explained the mechanisms and the patterns of adoption in order to assist in predicting whether and how an innovation will be successful. Depending on the readiness and willingness to adopt innovations, individuals or organizations can be categorized as:

- innovators (e.g. venturesome, enterprising),

- early adopters (e.g. social leaders, respectable),

- early majority (e.g. deliberate, considered),

- late majority (e.g. skeptical), or

- laggards (traditional).

Following [28] selection and adoption of technology takes the form of small, incremental, and continuous changes. These adjustments are cumulative, irreversible and specific to each production unit. Creating a website is the starting point for a firm to achieve the benefits derived from using the Internet [24]. The next, more advanced level in the use of the Internet is the establishment of Intranets and Extranets. According to Mendo and Fitzgerald (2005), depending on a firm's online strategy, its website will have different functional characteristics [23]. Although increasing numbers of businesses now have a website, there seems to be a quality problem that is leading to missed opportunities, particularly for smaller enterprises [27]. The design of a website needs to be viewed primarily as a business task, not a technical task. The website design needs to be appropriate to the needs of the company and should focus on supporting business goals. Supplier enablement is the channel that enterprises use to integrate with their trade partners and realize their e-sourcing and e-procurement activities [5]. The two main forms in that supplier enablement takes place in companies are through the application of supplier portals and/or e-marketplaces. In the case of supplier portals, they create the basis to connect suppliers with their buyers, with the focus on purchasing processes and the exchange of transactions data. They offer a structured and customized gateway to improve the business relations between two or more business partners. On the other side, e-marketplace is a virtual online market where buyers, suppliers, distributors and sellers find and exchange information, conduct trade, and collaborate with each other via an aggregation of information portals, trading exchanges and collaboration tools.

Skill accumulates around the technology the firm initially possessed, making it easier to adopt some types of new technologies rather than others. On the other hand, certain patterns of technical interaction (i.e., how information /knowledge is exchanged) are specific to different types of buyer-supplier relations (i.e., different types of organizational structures). Experimenting with a new technology is an important consideration for sophisticated adopters (usually early adopters) who must rely only upon the available information, while normal adopters (laggards) can learn from others' experience [12]. Besides, following [12] inter-firm differences in the patterns of adoption of ICT technologies may be due to the legacies of past technological investments more than to the availability of financial resources.

Costs, technological uncertainty and lack of relative advantage are the most important obstacles to ICT adoption. Costs refer not only to total investment, but also to the cost of information searches in relation to the new technology and the adaptation of existing networks to new standards. Empirical findings [12] suggest that in contexts where 
network effects matter, obstacles to adoption are eased by the development of compatible standards to encourage their adoption by SME.

Similar to Yun (1999) the primary objective is to understand how technological capabilities of small suppliers firms affect the nature of contracts. A broad interpretation of technological capability is defined to be the information and skills (technical, managerial and institutional) that allow suppliers to utilize ICT business tools efficiently. The contract determines the payment from the large firm to the supplier. For example, buyer-supplier relations that involve more than the exchange of price information are conducive to technology transfer to and learning by the suppliers. There have to be benefits for suppliers as well as for buyers. Both parts can cooperate closely in technical aspects and share investment in computer systems, marketing, logistics and payments. The potential benefits to small businesses can include reduced costs associated with inventories, procurement and distribution, increased productivity, improved profitability, improved quality of service, and secured competitive positions. There is a set of products and services for which the logistics costs are minor and especially suitable for online services. For example, consider sending money transfers online instead of sending checks offline.

\section{The Model}

Following [10] a large firm (principal) must choose among different suppliers (agents) to build a B2B electronic commerce network. The principal could be a large firm that decides to initiate electronic commerce with one of her suppliers. The large firm has to choose among her suppliers to introduce some ICT business tool for technology compatibility and provide them the right incentives to perform this task.

Compte and Jehiel(2008) main insight is that when several potential candidates compete for the task, the principal, in general, will improve the performance of her firm by inducing the member candidates to assess their competence before signing the contract (through an appropriate choice of contracts). The manager will (at least in some cases) be better off when candidates can more easily assess their competence, hence for example when he provides a more accurate description of the job to the agents.

In essence, their result follows from the following observation: when several agents are in competition for the contract, information acquisition accompanied by a proper screening device is socially desirable because it increases the chance that the principal will pick the most appropriate agent. A screening device is seen as a tool for selfselection to avoid adverse selection in situations of information asymmetry [25], where the less informed are able to distinguish the quality from the better informed by inscribing an autoselection process in their decisions. In this study, it is more likely that the manager will pick the most appropriate supplier firm in terms of technological compatibility to engage into the e-commerce network. In their setup, it is not merely the fact that there are several agents that drives the result. It also requires that private information bears on agents' competence (which is assumed to be agent specific) rather than on common characteristics that would apply equally to all agents. Because we talk about goods that have network externalities, the utility of the supplier will depend also on the network good.

The large firm attaches a value of $\mathrm{V}(\mathrm{q})$ to the adoption of $\mathrm{q}$ ICT business tools. In this model, suppliers are supposed to adopt different ICT business tools. Assume that $\mathrm{V}(0)=0$, and let $\mathrm{V}(1)=\mathrm{V}$ and $\mathrm{V}(2)=\mathrm{V}$.

Thus, $\mathrm{q}=0$, q1, q2.

- q1=1 means the supplier adopts a basic ICT business tool.

- q2=2 means the supplier adopts a superior ICT business tool.

This ordinal output assumes that ICT business tools are different in quality; thus, the principal or manager attaches a different value to the acquisition of them.

There are $\mathrm{n}$ suppliers, all of them have planned to incorporate ICT business tools and the chosen one will incorporate these technologies as a task. For each supplier i, the disutility of adopting an ICT business tool is equal to $\beta \mathrm{iq}$, where $\beta \mathrm{i}>0$. $\beta \mathrm{i}$ are the disutilities of technology adoption, each lower $\beta \mathrm{i}$ indicates that the supplier $\mathrm{i}$ fits the requirements better. It happens that some suppliers are more internet-oriented than others, such as a supplier with qualified employees and entrepreneur skills that foster a better technology adoption.

Utility is transferable. If qi ICT tools are introduced and the large firm pays $t$ to supplier $i$, the payoff to the large firm is $V(q)-t$ and the net benefit to supplier $i$ is $t-(\beta i-\gamma i) q$, where $y i$ is a parameter that reflects the preferences of the supplier over the network good. Because of ICT network externalities, there is an additional benefit for the supplier (additional to the monetary payment specified in a contract for non network goods) that comes from the ICT business tool adopted. Thus, the net benefit of the supplier depends negatively on the disutility of technology adoption and positively on the utility of the network good. When facing choices regarding how and whether to participate in such interactions as computer networks and telecommunications systems, one consideration is inevitably how our participation will affect others and how the participation of others will affect us.

The parameters of the problem are known to all parties, except for the disutilities of production, $\beta 1, \ldots \ldots, \beta n ;$ and the utilities for the network good $\gamma 1, \ldots \ldots . \mathrm{yn}$. We assume that each $\beta \mathrm{i}$ can take two possible values, $\beta_{i} \in\{\beta, \bar{\beta}\}, 0<\underline{\beta}<\bar{\beta}$. We assume that these parameters are drawn from identical and independent distributions. We let $\mathrm{p}$ denote the 
probability that supplier $\mathrm{i}$ is a low cost supplier: $p=\operatorname{Pr}\left\{\beta_{i}=\beta \underline{\beta}\right.$ and $(1-p)$ the probability of being a high cost supplier and assume that $0<p<1$.

Besides, each yi can take two possible values, $\gamma_{i} \in\{\gamma, \bar{\gamma}\}, 0<\underline{\gamma}<\bar{\gamma}$. Suppose there are only two types of suppliers, we call them sophisticated and normal, where the sophisticated ones benefit more both from the individual ICT business tool adoption and the network size. A normal supplier is mainly focused on the improvement of administrative and personnel issues of the specific organizational unit itself, thus leaving behind potential benefits of intra-organizational and inter-organizational collaboration. In terms of the adopter categories defined by[18], it can be characterized as laggard or late majority. The sophisticated supplier is rather focused on optimizing the supply chain (from supplier to the client). In terms of the adopter categories, it can be characterized as early adopter or innovator.

The probability of being a normal supplier is $\lambda=\operatorname{Pr}\left\{\gamma_{i}=\underline{\gamma}\right\}$ and $(1-\lambda)$ the probability of being a sophisticated supplier. Since these parameters are drawn from identical and independent distributions, we can construct the probability of the following events:

Probability of being a low cost and sophisticated supplier: $\operatorname{Pr}\left\{\beta_{i}=\beta ; \gamma_{i}=\bar{\gamma}\right\}=p \cdot \lambda=\mu$.

Probability of being a high cost and normal supplier: $\operatorname{Pr}\left\{\beta_{i}=\bar{\beta} ; \gamma_{i}=\underline{\gamma}\right\}=(1-p)(1-\lambda)=1-\mu$.

We suppose that if the supplier is high cost-sophisticated or low cost-normal, he will not adopt any technology ( $q=0)$. To understand this assumption, we argue that a low cost-normal supplier is indifferent between adopting the technology by contracting with the large firm or not, while the high cost- normal supplier is not, he prefers to adopt the technology by means of this contract. The high cost- normal supplier decides to introduce ICT tools to his business but he can not afford the acquisition and implementation costs. Therefore, he needs to contract with the large firm to acquire the ICT tool. The low cost- normal supplier, on the other hand, is indifferent between adopting the technology now or not. If he had preferred adopting the technology, he would have done it before signing the contract, since his costs are low enough to adopt the technology by himself. However, he decides not to adopt the ICT technology now. The supplier knows he can adopt it in the future; 'being first' is not an important goal for him. This explanation does not apply to the low cost-sophisticated supplier, who will never be indifferent between adopting and not adopting the ICT due to his preferences: he always prefers adopting the technology. Because he is sophisticated, he will prefer compatibility with the large firm and the future suppliers. Hence, he will always prefer adopting ICT business tools and complementary products. However, for a high cost sophisticated supplier adopting the technology is not a profitable decision.

The social surplus is equal to $E S=V\left(q_{i}\right)-\left(\beta_{i}-\gamma_{i}\right) q_{i}$

We assume that for a low cost and sophisticated supplier, the social surplus is largest when a superior ICT tool is adopted, and that for a high cost and normal supplier, the social surplus is largest when a basic ICT tool is adopted, that is:

$$
\begin{aligned}
& \text { For } \underline{\beta}, \bar{\gamma}, E S=V-2(\underline{\beta}-\bar{\gamma}) \\
& \text { If } V-2(\underline{\beta}-\bar{\gamma})>v-(\underline{\beta}-\bar{\gamma}) \text {, then } V-v>(\underline{\beta}-\bar{\gamma}) \\
& \text { For } \bar{\beta}, E S=v-(\bar{\beta}-\underline{\gamma}) \\
& \text { If } v-(\bar{\beta}-\underline{\gamma})>V-2(\bar{\beta}-\underline{\gamma}) \text {, then }(\bar{\beta}-\underline{\gamma})>V-v \\
& \text { Thus, }(\underline{\beta}-\bar{\gamma})<V-v<(\bar{\beta}-\underline{\gamma}) ;(\underline{\beta}-\bar{\gamma})<(\bar{\beta}-\underline{\gamma})
\end{aligned}
$$

After signing a contract, the supplier will learn at no cost if he is high cost or low cost. In this setting, if the supplier observes $\beta$ immediately after being offered a contract (but yet not signed) it will be at positive cost (c>0) reflecting the difference in cost between acquiring information in the precontractual and postcontractual phases, the weight of the principal assistance over the cost of acquiring supplier type information.

The objective of the paper is to expand Compte and Jehiel's results in a network effects setting based on [1], to show how the presence of different types of suppliers alters the large firm's inducing information acquisition contracts.

\subsection{Results}

Following [10], consider the case in which the large firm would induce no information acquisition. In this case, suppliers are identical ex ante. Thus, it is irrelevant which one is selected. The maximum surplus ES generated by any match is then obtained when the supplier adopts a specialized ICT business tool if low cost-high utility and a basic one, if high cost. Then, 


$$
E S=\mu(V-2(\underline{\beta}-\bar{\gamma}))+(1-\mu)(v-(\bar{\beta}-\underline{\gamma}))
$$

Compared to the maximum surplus obtained in a non network goods setting (put $\mathrm{\gamma}=0$ ), this one is increased by the amount $2 \mu(\bar{\gamma}-\underline{\gamma})+\underline{\gamma}$. Then, the more sophisticated the supplier is, the larger the expected surplus.

Since the suppliers have the option to refuse any contractual offer, they cannot obtain an expected payoff below 0 . Thus, ES is an upper bound on the payoff obtained by the large firm when she does not induce information acquisition.

Proposition I. The expected payoff obtained by the large firm if she does not induce information acquisition is at most equal to $E S$.

According to [13], the payoff obtained by the large firm will be strictly smaller than ES, because of the incentives she has to give suppliers not to acquiring information before signing the contract.

Then, [10] turn to the case in which the principal induces information acquisition. It is assumed that the principal makes a sequence of contractual offers to agent $1,2, \ldots, n$, until one agent accepts.

Proposition 2 generates the same results as in the case of non network goods, the supplier prefers acquiring information. The supplier's payoff specified in contract $\mathrm{C} 1$ add the utility over the network good.

Proposition II. Assume that the large firm always offers the contract C1, defined as follows: "Introduce a superior ICT tool and receive a transfer equal to $T=2(\underline{\beta}-\bar{\gamma})+c^{+} / \mu$ ", where $c^{+}$means a slightly positive information acquisition cost. There exists $c^{\prime}>0$ such that if $c<c^{\prime}$, then (a) any supplier who is offered this contract acquires information and accepts the contract if and only if he is a low cost-sophisticated supplier, and (b) expected payoff to the large firm exceeds ES if $\mathrm{n}$ is large enough and $\bar{\gamma}>0$.

Proof. The presence of utility over network goods introduces an additional constraint on the large firm's expected payoff to be larger than the expected surplus ( $n$ large is not a sufficient condition).

To prove proposition II, observe that a high cost-normal supplier will not accept the contract, since $T<2(\bar{\beta}-\underline{\gamma})+c / \mu$.

If the supplier does not acquire information about his type and still signs the contract he gets $T-2 E(\beta-\gamma)$ which is negative for $\mathrm{c}$ small enough.

Supplier expected payoff $=\mu(T-2(\underline{\beta}-\bar{\gamma}))+(1-\mu)(T-2(\bar{\beta}-\underline{\gamma}))=T-2(\mu(\underline{\beta}-\bar{\gamma})-\mu(\bar{\beta}-\underline{\gamma})+(\bar{\beta}-\underline{\gamma}))=$ $T-2 \mu(\underline{\beta}-\bar{\gamma}-(\bar{\beta}-\underline{\gamma}))-2(\bar{\beta}-\underline{\gamma}) T-2(\mu(\underline{\beta}-\bar{\gamma})+(1-\mu)(\bar{\beta}-\underline{\gamma}))=T-2(E \beta-E \gamma)=T-2 E(\beta-\gamma) \square$

Following [8] the large firm's expected payoff is equal to

$\left(1-(1-\mu)^{n}\right)(V-T)$

Which exceeds ES when $\mathrm{n}$ is large enough and $\mathrm{c}$ small enough and if the large firm internalizes the externality. $\left(1-(1-\mu)^{n}\right)(V-T)$ converges to $V-2(\underline{\beta}-\bar{\gamma})$ as $n \rightarrow \infty, c \rightarrow 0$ and because $E S<V-2(\underline{\beta}-\bar{\gamma})$, since $(\underline{\beta}-\bar{\gamma})<(\bar{\beta}-\underline{\gamma})$.

To prove that $E S<V-2(\underline{\beta}-\bar{\gamma})$, assume $\mu(V-2(\underline{\beta}-\bar{\gamma}))+(1-\mu)(v-(\bar{\beta}-\underline{\gamma}))>V-2(\underline{\beta}-\bar{\gamma})$.

Rearranging this expression we get $(\underline{\beta}-\bar{\gamma})-(V-v)>(\bar{\beta}-\underline{\gamma})-(\underline{\beta}-\bar{\gamma})$. This cannot be true since the left side of this inequality is negative. Following the same reasoning, $E S=V-2(\underline{\beta}-\bar{\gamma})$ is false. Thus, $E S<V-2(\beta-\bar{\gamma})$, the expected payoff to the principal exceeds ES.

\section{Second Best}

Proposition II shows that inducing some information acquisition is good for the large firm. To get the optimal contract, utilities over the network good are introduced, so that the expected payoff obtained by the large firm under the optimal contract is a function of the information acquisition and the network externality.

Following [10] we define cand $\overline{c^{*}}$, let $\underline{\mathrm{c}}=\mu(1-\mu)(\bar{\beta}-\underline{\beta})$ and $\bar{c}^{*}=\mu(1-\mu)[V-2(\underline{\beta}-\bar{\gamma})-(v-(\bar{\beta}-\underline{\gamma})]$ and $\underline{c}<\overline{c^{*}}$ as $V-v>\underline{\beta}-\bar{\gamma}$. 
Define a new contract C2 as follows: "Either introduce a basic ICT tool for a transfer $\mathrm{t}=\mathrm{E} \beta-\mathrm{E} \gamma$, or a superior ICT tool for a transfer $\mathrm{T}=\mathrm{t}+\beta(+\varepsilon)$.

Proposition III. Assume that $c \in\left(c, \bar{c}^{*}\right), \mathrm{n}=2$ and their definitions of $\underline{c}$ and $\bar{c}^{*}$. The optimal contract can be implemented by offering $C 1$ to supplier 1 and contract $C 2$ to supplier 2 in case supplier 1 rejects $C 1$. The optimal contract yields to the large firm an expected payoff equal to $E S+\bar{C}^{*}-C$

Proof.

Step 1: The large firm cannot get more than $E S+\bar{C}^{*}-C$

The best economic outcome is that when one of the two suppliers is a low cost-sophisticated one and he is selected to adopt a superior ICT, and otherwise adopt a basic one. The first event has probability $\mu+(1-\mu) \mu$, and if the supplier acquires information, then the corresponding expected surplus is given by:

$[\mu+(1-\mu) \mu][V-2(\underline{\beta-\bar{\gamma}})]+(1-\mu)^{2}[v-(\bar{\beta}-\gamma)]$ which equals $E S+\bar{c}^{*}$. Where ES is the highest surplus obtained with one supplier or without information acquisition.

$$
\begin{aligned}
& {[\mu+(1-\mu) \mu]\left(V-2(\underline{\beta}-\bar{\gamma})+(1-\mu)^{2}(v-\bar{\beta}-\underline{\gamma})=\mu\left(V-2(\underline{\beta}-\bar{\gamma})+(1-\mu) \mu\left(V-2(\underline{\beta}-\bar{\gamma})+\left(1-2 \mu+\mu^{2}\right)(v-(\underline{\beta}-\bar{\gamma})=\right.\right.\right.} \\
& \mu(V-2(\underline{\beta}-\bar{\gamma}))+(1-\mu) \mu(V-2(\underline{\beta}-\bar{\gamma}))+(1-\mu)(v-(\bar{\beta}-\underline{\gamma}))-\mu(1-\mu)(v-(\bar{\beta}-\underline{\gamma}))=E S+\bar{c}^{*} \square
\end{aligned}
$$

When one supplier acquires information the maximum surplus net of information acquisition costs is thus equal to $E S+\bar{C}^{*}-c$, which is the maximum expected payoff the large firm can hope to get.

Step 2.

When $c \in\left(c, \bar{c}^{*}\right)$, the contract $\mathrm{C} 2$ yields the large firm an expected payoff equal to $\mathrm{ES}$.

Proof. By construction we have:

$T-2(\underline{\beta}-\bar{\gamma})>t-(\underline{\beta}-\bar{\gamma})$ and $T-2(\bar{\beta}-\underline{\gamma})<t-(\underline{\beta}-\bar{\gamma})$. This means $\underline{\beta}-\bar{\gamma}<T-t<\bar{\beta}-\underline{\gamma}$.

Hence a supplier who would accept this contract without acquiring information would adopt a superior ICT if low costsophisticated, and a basic ICT if high cost-normal. Then, he would obtain an expected payoff G supplier that satisfies

G supplier $=\mu(T-2(\underline{\beta}-\bar{\gamma}))+(1-\mu)(t-(\bar{\beta}-\underline{\gamma}))$

This is positive by construction.

However, if $c \notin\left(\underline{c}, \bar{c}^{*}\right)$ and $c>\bar{c}^{*}$ were the case, we would have $E \beta>\bar{\beta}-c / 1-\mu$. Hence we would have

$t>\bar{\beta}-c / 1-\mu, \mu-\bar{\beta}>-c /(1-\mu),(1-\mu)(\mu-\bar{\beta})>-c=G^{\text {sup plier }}-\mu(T-2 \underline{\beta})$. Then $G^{\text {sup } p l i e r}-\mu(T-2 \underline{\beta})>-c$,

so we would also have $\mathrm{G}$ supplier $>\mu(T-2(\underline{\beta}-\bar{\gamma}))-c$. As the supplier's expected payoff is decreasing on $\mathrm{c}$, he would prefer signing without acquiring information on its competence.

The expected payoff that the large firm obtains is $E S-G^{\text {sup plier }}$

G large firm $=E S-G^{\text {sup plier }}=\mu(V-2 \beta)+(1-\mu)(v-\bar{\beta})-\mu(T-2 \beta)-(1-\mu)(t-\bar{\beta})$

G large firm $=\mu(V-T)+(1-\mu)(v-t)$

Step 3. When the large firm offers contracts as in proposition III she obtains an expected payoff equal to $E S+\overline{C^{*}}-c$.

Proof. When the large firm offers $\mathrm{C} 1$ to supplier 1 , supplier 1 acquires information and rejects the contract in the event he is a high cost supplier. In the latter case, she offers C2 to supplier 2, and obtains an expected payoff equal to ES. Overall, her expected payoff is equal to

G large firm $=\mu(V-T)+(1-\mu) E S$

G large firm $=\mu(V-2(\underline{\beta}-\bar{\gamma}))-c+(1-\mu) E S$

Since $\overline{c^{*}}=\mu(V-2(\underline{\beta}-\bar{\gamma})-E S), \mu(V-2(\underline{\beta}-\bar{\gamma}))=\mu E S+\bar{C}^{*}$ 
G large firm $=\mu E S+\bar{c}-c+(1-\mu) E S=E S+\bar{c}^{*}-c$

\section{Conclusion}

The application of information technology and the formation of electronic networks have become an important part of corporate strategy. This statement is especially true in the management of company's relationship with their suppliers. There has been a growing body of qualitative research into the use of ecommerce but the focus has tended to be on the larger firm, on developing new business models and positioning its development in the new economy. Small firms have been much slower to adopt e-business and relevant research has also been slow to develop. One of the main problems of understanding the needs of the small firm is the fact that they are not a homogeneous group and so their needs will vary by sector. High-technology and knowledge intensive small firms are more likely to use e-commerce than other small firms and there are differences between industry sectors in terms of e-commerce use and strategy development. Opportunities include the ability to reduce transaction costs, the development of a more level playing field with larger firms, being able to extend marketing efforts, improve communications, identify and develop new markets, cost reduction and developing relationships with suppliers.

In this paper we model how the implementation of e-commerce technologies could change the relationship between a large buyer and her suppliers. Usually, e-commerce implementations are initiated by the more powerful partner in a relationship, commonly the buyer. The success of many electronic commerce B2B operations depends on the ability to manage data and transform it into information. This ability concerns not just to a single firm but to the members of a supply chain. E-commerce implementation by a large firm, leader of a supply chain, requires compatibility and interoperability of her suppliers, among other things. Yet the suppliers play an important role in a supply chain ICT implementation from a technology and business perspective. Since B2B e-commerce involves conducting B2B transactions through Internet websites, many suppliers must implement these technologies either by themselves or by a large partner incentive mechanism.

The productivity gains from B2B that foster E-commerce growth come from the return to ICT business tools investments and from network externalities. In the model, a large firm is planning doing B2B with some of her SME suppliers. Thus, the contracted supplier's task consists in the adoption of some ICT business tool. For instance, the supplier can incorporate to its website the ability to buy by mail order or additionally to accept online payments through an automated process. The results of the paper follow directly from [1] and [10]. Once network effects are introduced to the model, an increase in social surplus takes place. When several potential SME suppliers compete for ICT modernization, the large firm will in general improve the performance of her firm by inducing the suppliers to assess their competence before signing the contract through an appropriate choice of contracts that consider the presence of different types of suppliers in terms of technology adoption costs and preferences over the technology.

This model applies better to contexts where the incentives to suppliers ICT implementation by large manufacturers are more significant than the initiatives of outsourcing firms because buyers tend to have more control on the supply chain. Their low level of outsourcing may be due to the fact that their products are less standardized and therefore more complex to outsource. This model takes as given the large firm's decision on e-commerce investment. This decision is exogenous in the model. We do not analyze the large firm's first decision: whether to invest on Ecommerce or not. We focus on the consequences of e-commerce implementation from a social view point. Therefore, a possible extension of this paper could be to introduce the large firm's decision to invest on e-commerce.

In contexts where (physical) network effects matter, it is important to maintain backward compatibility. Therefore, in order to speed up adoption of new technologies, one would need to stimulate the development of compatible and/or interoperable standards in the market. Thus, the diffusion of information about the benefits and costs of ICT business tools as well as the reduction of uncertainty from a large business partner could be a feasible way to promote SME ICT adoption. Besides, not always the provision of generic public funds or other types of financing instruments for ICT adoption by SME are supply chain incentive compatible.

\section{References}

[1] M. V. Alderete, E-commerce between a large firm and a SME supplier: A screening model, Journal of Computing, vol. 1, no. 1, pp. 158-161, 2009.

[2] J. Y. Bakos, Information links and electronic marketplaces: The role of interorganizational information systems in vertical markets, Journal of Management Information Systems, vol. 8, no. 2, pp. 31-52, 1991.

[3] E. Baraldi and G. Nadin, The challenges in digitalising business relationships. The construction of an IT infrastructure for a textile-related business network, Technovation, vol. 26, no. 10, pp. 1111-1126, 2006.

[4] A. Barua, P. Konana, A. Whinston, and F. Yin, Making e-Business pay: Eight key drivers for operational success, IEEE IT Professional, vol. 2, no. 6, pp. 2-10, 2000.

[5] E. G. Campelo and W. Stucky, The supplier relationship management trends, World Academy of Science, Engineering and Technology, vol. 28, pp. 105-110, April 2007. 
[6] M. Clements, Direct and indirect network effects: are they equivalent?, International Journal of Industrial Organization, vol. 22, no. 5, pp. 633-645, 2004.

[7] S. Chong, Success in electronic commerce implementation: A cross country study of small and medium sized enterprises, Journal of Enterprise Information Management, vol. 21, no. 5, pp. 468-492, 2008.

[8] S. Chong, An empirical study of factors that influence the extent of deployment of electronic commerce for small and medium sized enterprises in Australia, Journal of Theoretical and Applied Electronic Commerce Research, vol. 1 , no. 2 , pp. 45-57, 2006

[9] J. Church and N. Gandal, Networks effects, software provision and standardization, The Journal of Industrial Economics, vol. 4, no. 1, pp. 85-103, 1992.

[10] O. Compte and P. Jehiel, Gathering information before signing a contract: A screening perspective, International Journal of Industrial Organization, vol. 26, no.1, pp. 206-212, 2008.

[11] N. Corrocher and R. Fontana, Expectations, network effects and timing of technology adoption: Some empirical evidence from a sample of SMEs in Italy, Small Business Economics, vol. 31, no. 4, pp. 425-441, 2006.

[12] N. Corrocher and R. Fontana, Objectives, obstacles and drivers of ICT adoption: What do IT managers perceive?, Information Economics \& Policy, vol. 20, no. 3, pp. 229-242, 2008.

[13] J. Crémer and F. Khalil, Gathering information before signing a contract, American Economic Review, vol. 82, no. 3, pp. 566-578, 1992.

[14]R. R. Dholakia and N. Kshetri, Factors Impacting the Adoption of the Internet among SMEs, Small Business Economics, vol. 23, no. 4, pp. 311-322, 2004

[15] Z. Fernández and M. J. Nieto, The internet: strategy and boundaries of the firm, Business Economics Series Working Paper, vol. 1, no. 1, pp. 1-23, 2005.

[16]E. Grandon and J. M. Pearson, Electronic commerce adoption: an empirical study of small and medium US businesses, Information \& Management, vol. 42, no. 1, pp. 197-216, 2003.

[17] M. L. Katz and C. Shapiro, Systems competition and network effects, The Journal of Economic Perspectives, vol 8, no. 2, pp. 93-115, 1994.

[18] L. A. Lefebrve, E. Lefebrve, E. Elia, and H. Boeck, Exploring B-to-B e-commerce adoption trajectories in manufacturing SMEs, Technovation, vol. 25, no. 12, pp. 1443-1456, 2005.

[19] T. Mettler and P. Rohner, E-procurement in hospital pharmacies: An exploratory multicase study from Switzerland, Journal of Theoretical and Applied Electronic Commerce Research, vol. 4, no. 1, pp. 23-38, 2009.

[20] OECD. ICT, E-Business and SMEs. OECD, Paris, 2004.

[21] H. Schaffers, K. Gareis, K. Stanoevska-Slabeva, M. Vartiainen, and M. Bijlsma, The role of collaborative working environments in enabling global businesses, in Proceedings of International Conference on Concurrent Enterprising ICE, Lisboa, Portugal, 2008, pp. 1-8.

[22] G. P. Schneider, Electronic Commerce, Eighth edition. Boston: Cengage Learning, 2009.

[23] G. Simmons, G. Armstrong, and M. Durkin, A conceptualization of the determinants of small business website adoption: Setting the research agenda, International Small Business Journal, vol. 26, no. 4, pp. 351-389, 2008.

[24] P. Soto-Acosta and A. Meroño-Cerdan, Evaluating internet technologies business effectiveness, Telematics and Informatics, vol. 26, no. 2, pp. 211-221, 2009.

[25] M. Spence, Signaling in retrospect and the informational structure of markets, The American Economic Review, vol. 92, no. 3, pp. 434-459, pp. 149-159, 2002.

[26] M. Thelwall, Effective websites for small and medium-sized enterprises, Journal of Small Business and Enterprise Development, vol. 7, no. 2, pp.149-159, 2000.

[27] UNCTAD, Science and technology for development: the new paradigm of ICT, Information economy report 2007-2008, 2008.

[28] M. Yun, Subcontracting relations in the Korean automotive industry: Risk sharing and technological capability, International Journal of Industrial Organization, vol. 17, no. 1, pp. 81-108, 1999.

[29] B.A. Wagner, I. Fillis, and U. Johansson, E-business and e-supply strategy in small and medium sized businesses (SMEs), Supply Chain Management: An International Journal, vol. 8, n. 4, pp. 343-354, 2003.

[30] K. Zhu, K. Kraemer, and S. Xu, E-Business adoption by European firms: A cross-country assessment of the facilitators and inhibitors, European Journal of Information Systems, vol. 12, no. 4, pp. 251-268, 2003. 